\title{
On the uniform theory of lacunary series
}

\author{
I. Berkes*
}

Dedicated to Professor Robert Tichy on the occasion of his 60th birthday

\begin{abstract}
The theory of lacunary series starts with Weierstrass' famous example (1872) of a continuous, nondifferentiable function and now we have a wide and nearly complete theory of lacunary subsequences of classical orthogonal systems, as well as asymptotic results of thin subsequences of general function systems. However, many applications of lacunary series in harmonic analysis, orthogonal function theory, Banach space theory, etc. require uniform limit theorems for such series, i.e. theorems holding simultaneously for a class of lacunary series, and such results are much harder to prove than dealing with individual series. The purpose of this paper is to give a survey of uniformity theory of lacunary series and discuss new results in the field. In particular, we study the permutation-invariance of lacunary series and their connection with Diophantine equations, uniform limit theorems in Banach space theory, resonance phenomena for lacunary series and lacunary series with random gaps.
\end{abstract}

\section{Introduction}

Let $\left(n_{k}\right)$ be a sequence of positive integers satisfying the Hadamard gap condition

$$
n_{k+1} / n_{k} \geq q>1 \quad(k=1,2, \ldots) .
$$

Salem and Zygmund [47] proved that $\left(\cos 2 \pi n_{k} x\right)$ obeys the central limit theorem

$$
\lim _{N \rightarrow \infty} \lambda\left\{x \in(0,1):(N / 2)^{-1 / 2} \sum_{k=1}^{N} \cos 2 \pi n_{k} x \leq t\right\}=(2 \pi)^{-1 / 2} \int_{-\infty}^{t} e^{-u^{2} / 2} d u
$$

where $\lambda$ denotes the Lebesgue measure. Under the same gap condition Erdös and Gál [27] proved that $\left(\cos 2 \pi n_{k} x\right)$ obeys the law of the iterated logarithm

$$
\limsup _{N \rightarrow \infty}(N \log \log N)^{-1 / 2} \sum_{k=1}^{N} \cos 2 \pi n_{k} x=1 \quad \text { a.e. }
$$

\footnotetext{
*Graz University of Technology, Institute of Statistics, Kopernikusgasse 24, 8010 Graz, Austria. e-mail: berkes@tugraz.at. Research supported by FWF grant P24302-N18.
} 
These results show that under the gap condition (1.1) the functions $\cos 2 \pi n_{k} x$ behave like independent random variables. Morgenthaler [43] and Weiss [52] showed that the analogue of (1.2) and (1.3) holds for any uniformly bounded orthonormal system except that one needs a much stronger gap condition for $\left(n_{k}\right)$, the Gaussian limit distribution in (1.2) becomes mixed Gaussian and the limsup in (1.3) becomes a nonconstant function of $x$. As shown by Gaposhkin [32], at the cost of introducing an extra centering factor, even the orthogonality can be dropped here. Specifically, he proved (see also Chatterji [23], [24]) that if a sequence $\left(X_{n}\right)$ of r.v.'s satisfies $\sup _{n} E X_{n}^{2}<\infty$, then one can find a subsequence $\left(X_{n_{k}}\right)$ and r.v.'s $X$ and $Y \geq 0$ such that

$$
\frac{1}{\sqrt{N}} \sum_{k \leq N}\left(X_{n_{k}}-X\right) \stackrel{d}{\longrightarrow} N(0, Y)
$$

and

$$
\limsup _{N \rightarrow \infty} \frac{1}{\sqrt{2 N \log \log N}} \sum_{k \leq N}\left(X_{n_{k}}-X\right)=Y^{1 / 2} \quad \text { a.s. }
$$

where $\stackrel{d}{\longrightarrow}$ denotes convergence in distribution and $N(0, Y)$ denotes the distribution of the r.v. $Y^{1 / 2} \zeta$ where $\zeta$ is a standard normal r.v. independent of $Y$. Komlós [40] proved that under $\sup _{n} E\left|X_{n}\right|<\infty$ there exists a subsequence $\left(X_{n_{k}}\right)$ and an integrable r.v. $X$ such that

$$
\lim _{N \rightarrow \infty} \frac{1}{N} \sum_{k=1}^{N} X_{n_{k}}=X \quad \text { a.s. }
$$

and Chatterji [22] showed that under $\sup _{n} E\left|X_{n}\right|^{p}<\infty, 0<p<2$ the conclusion of the previous theorem can be changed to

$$
\lim _{N \rightarrow \infty} \frac{1}{N^{1 / p}} \sum_{k=1}^{N}\left(X_{n_{k}}-X\right)=0 \quad \text { a.s. }
$$

for some $X$ with $E|X|^{p}<\infty$. Relations (1.4), (1.5), (1.6), (1.7) are the analogues of the central limit theorem, law of the iterated logarithm, strong law of large numbers and Marczinkiewicz's strong law, respectively, in a slightly modified form. Note the randomization in all these examples: the role of the mean and variance of the subsequence $\left(X_{n_{k}}\right)$ is played by random variables $X, Y$. On the basis of these and several other examples, Chatterji [25] formulated the following heuristic principle:

Subsequence Principle. Let $T$ be a probability limit theorem valid for all sequences of i.i.d. random variables belonging to an integrability class $L$ defined by the finiteness of a norm $\|\cdot\|_{L}$. Then if $\left(X_{n}\right)$ is an arbitrary (dependent) sequence of random variables satisfying $\sup _{n}\left\|X_{n}\right\|_{L}<+\infty$ then there exists a subsequence $\left(X_{n_{k}}\right)$ satisfying $T$ in a randomized form.

In a profound paper, Aldous [7] proved the validity of this principle for all distributional and almost sure limit theorems for i.i.d. random variables, subject to mild technical conditions. For a precise formulation, we refer to [7]. This result 
closes a long series of investigations and, together with a wide asymptotic theory of "concrete" (e.g. Hadamard lacunary) subsequences of classical function systems such as the trigonometric and Walsh system, orthogonal polynomials, dilated series $\sum c_{k} f(k x)$ (for a survey see Gaposhkin [32]), gives a quite satisfactory picture of the asymptotic behavior of lacunary series. Despite this fact, some important question in the field remain open. By a classical result of Menshov [42], every orthornormal system $\left(\varphi_{n}\right)$ contains a subsequence $\left(\varphi_{n_{k}}\right)$ which is a convergence system, i.e. under $\sum_{k=1}^{\infty} c_{k}^{2}<\infty$ the series $\sum_{k=1}^{\infty} c_{k} \varphi_{n_{k}}$ converges almost everywhere. Since every sequence of independent random variables with mean 0 and variance 1 has this property, for every fixed $\left(c_{k}\right)$ with $\sum_{k=1}^{\infty} c_{k}^{2}<\infty$, Aldous' theorem guarantees the existence of a subsequence $\left(\varphi_{n_{k}}\right)$ such that $\sum_{k=1}^{\infty} c_{k} \varphi_{n_{k}}$ converges a.e., but the subsequence $\left(n_{k}\right)$ depends on the coefficients $c_{k}$ and the existence of a subsequence having the above convergence property simultaneously for all $\left(c_{k}\right)$ does not follow. A related famous problem of the theory of orthogonal sequences (see Uljanov [51], p. 48)) was if every orthonormal sequence $\left(\varphi_{n}\right)$ has a subsequence which is a convergence system after any rearrangement of its terms. For a specific permutation of its terms, this follows again from Menshov's theorem, but whether there exists a subsequence $\left(\varphi_{n_{k}}\right)$ having this property simultaneously after all rearrangements remained open until settled by Komlós [41]. By a classical result of Kadec and Pełczyński [37], every normalized weakly null sequence in $L^{p}, p>2$ contains a subsequence spanning a subspace isomorphic to $\ell^{2}$ or $\ell^{p}$ and another profound result of Aldous [8] states that every closed infinite dimensional subspace $H$ of $L^{1}$ contains a subspace isomorphic to $\ell^{p}$ for some $1 \leq p \leq 2$. Equivalently, these results concern the behavior of norms $\left\|\sum_{k=1}^{n} c_{k} f_{n_{k}}\right\|$ for lacunary sequences $\left(f_{n_{k}}\right)$ in $L^{p}, p>2$ or in $H$ with the additional requirement that the estimates must be uniform for all $\left(c_{1}, \ldots, c_{k}\right) \in \mathbb{R}^{k}, k=1,2, \ldots$. Since an i.i.d. sequence is a symmetric structure, from the subsequence principle one would expect that limit theorems for lacunary series are permutation-invariant but, as we will see, this is not the case and establishing permutation-invariance of series $\sum c_{k} f_{n_{k}}$ requires considerable efforts. Note that permutation-invariance of lacunary series is also a uniformity problem, requiring the study of series $\sum c_{k} f_{\sigma(k)}$ simultaneously for all permutations $\sigma$ of $\mathbb{N}$. Finally, studying trigonometric series

$$
\sum_{k=1}^{\infty}\left(a_{k} \cos n_{k} x+b_{k} \sin n_{k} x\right)
$$

with random frequencies $n_{k}$ (such series provide an important tool, e.g., for constructing counterexamples in analysis) also requires studying a class of trigonometric series simultaneously, namely for all realizations $\left(n_{k}(\omega)\right), \omega \in \Omega$ in the underlying probability space $(\Omega, \mathcal{F}, P)$. The purpose of the present paper is to give a general study of such uniformity problems and formulate recent results in the field. 


\section{Limit random measure and a structure the- orem}

Using the terminology of [15], we call a sequence $\left(X_{n}\right)$ of random variables determining if it has a limit distribution relative to any set $A$ in the probability space with $P(A)>0$, i.e. for any $A \subset \Omega$ with $P(A)>0$ there exists a distribution function $F_{A}$ such that

$$
\lim _{n \rightarrow \infty} P\left(X_{n} \leq t \mid A\right)=F_{A}(t)
$$

for all continuity points $t$ of $F_{A}$. By an extension of the Helly-Bray theorem (see [15]), every tight sequence of r.v.'s contains a determining subsequence. As is shown in [7], [15], for any determining sequence $\left(X_{n}\right)$ there exists a random measure $\mu$ (i.e. a measurable map from the underlying probability space $(\Omega, \mathcal{F}, \mathcal{P})$ to the class $\mathcal{M}$ of probability measures on $\mathbb{R}$ equipped with the Lévy metric) such that for any $A$ with $P(A)>0$ and any continuity point $t$ of $F_{A}$ we have

$$
F_{A}(t)=\mathbb{E}_{A}(\mu(-\infty, t])
$$

where $\mathbb{E}_{A}$ denotes conditional expectation given $A$. We call $\mu$ the limit random measure of $\left(X_{n}\right)$. Let $\left(Y_{n}\right)$ be a sequence of random variables, defined on the same probability space, conditionally i.i.d. with respect to their tail $\sigma$-field $\mathcal{F}_{\infty}$ with conditional distribution $\mu$. (For the construction of such an $\left(Y_{n}\right)$ one may need to enlarge the probability space.) Clearly, $\left(Y_{n}\right)$ is exchangeable (i.e. for any permutation $\sigma: \mathbb{N} \rightarrow \mathbb{N}$ of the positive integers, the sequence $\left(Y_{\sigma(n)}\right)$ has the same distribution as $\left.\left(Y_{n}\right)\right)$ and satisfies limit theorems of i.i.d. random variables in a mixed form. For example, if $E Y_{1}^{2}<\infty$ and $Y=E\left(Y_{1} \mid \mathcal{F}_{\infty}\right), Z=\operatorname{Var}\left(Y_{1} \mid \mathcal{F}_{\infty}\right)$, then

$$
N^{-1 / 2} \sum_{k=1}^{N}\left(Y_{k}-Y\right) \stackrel{d}{\longrightarrow} N(0, Z)
$$

and

$$
\limsup _{N \rightarrow \infty}(2 N \log \log N)^{-1 / 2} \sum_{k=1}^{N}\left(Y_{k}-Y\right)=Z^{1 / 2} \quad \text { a.s. }
$$

If $E\left|Y_{1}\right|<\infty$ and $Y=E\left(Y_{1} \mid \mathcal{F}_{\infty}\right)$, then

$$
\lim _{N \rightarrow \infty} N^{-1} \sum_{k=1}^{N} Y_{k}=Y \quad \text { a.s. }
$$

We call $\left(Y_{n}\right)$ the limit exchangeable sequence of $\left(X_{n}\right)$. Aldous' subsequence theorem, mentioned in the Introduction, states that if $\left(X_{n}\right)$ is a tight sequence of random variables with limit random measure $\mu$, then sufficiently thin subsequences of $\left(X_{n}\right)$ satisfy all limit theorems valid for $\left(Y_{n}\right)$. To make this precise, one has to formalize the concept "limit theorem", which is rather technical, for the details we refer to Aldous [7].

The simplest exchangeable sequences are finite mixtures of i.i.d. sequences, i.e. sequences $\left(X_{n}\right)$ for which there exists a finite partition $\Omega=\cup_{j=1}^{r} B_{j}$ of the underlying 
probability space such that, restricted to any $\left(B_{j}\right),\left(X_{n}\right)$ is an i.i.d. sequence. The following theorem, proved by Berkes and Péter [13], shows that given a tight sequence $\left(X_{n}\right)$ of random variables, sufficiently thin subsequences $\left(X_{n_{k}}\right)$ can be approximated arbitrary closely by finite mixtures of i.i.d. sequences. This theorem yields an immediate, simple way to prove the subsequence principle and it will be the main tool for the proof of our results discussed in later chapters.

Theorem 2.1 Let $\left(X_{n}\right)$ be a determining sequence of r.v.'s and $\left(\varepsilon_{n}\right)$ a positive numerical sequence. Then there exists a subsequence $\left(X_{m_{k}}\right)$ and a sequence $\left(Y_{k}\right)$ of discrete r.v.'s such that

$$
P\left(\left|X_{m_{k}}-Y_{k}\right| \geq \varepsilon_{k}\right) \leq \varepsilon_{k} \quad k=1,2 \ldots
$$

and for each $k>1$ the atoms of the finite $\sigma$-field $\sigma\left\{Y_{1}, \ldots, Y_{k-1}\right\}$ can be divided into two classes $\Gamma_{1}$ and $\Gamma_{2}$ such that

(i) $\sum_{B \in \Gamma_{1}} P(B) \leq \varepsilon_{k}$

(ii) For any $B \in \Gamma_{2}$ there exist $P_{B}$-independent r.v.'s $\left\{Z_{j}^{(B)}, j=k, k+1, \ldots\right\}$ defined on $B$ with common distribution function $F_{B}$ such that

$$
P_{B}\left(\left|Y_{j}-Z_{j}^{(B)}\right| \geq \varepsilon_{k}\right) \leq \varepsilon_{k} \quad j=k, k+1, \ldots
$$

Here $F_{B}$ denotes the limit distribution of $\left(X_{n}\right)$ relative to $B$ and $P_{B}$ denotes conditional probability given $B$.

\section{Permutation-invariance}

Studying rearrangements of function series $\sum_{k=1}^{\infty} f_{k}$ is a much investigated problem of analysis. By the Riemann rearrangement theorem, a conditionally convergent numerical series $\sum_{k=1}^{\infty} c_{k}$ can be rearranged to converge to any prescribed real number and to $+\infty$ and $-\infty$. The corresponding problem for function series, called Banach's problem, is much harder and is still unsolved. (See Nikishin [45], Theorem 22 for the strongest existing result.) Another famous conjecture of analysis is that any orthonormal system $\left(\varphi_{n}\right)$ can be rearranged to become a convergence system. (See Garsia [35] for a partial result.) Unconditional a.e. convergence (i.e. almost everywhere convergence after every rearrangement) of function series is also an important topic in functional analysis. Lacunary series play a special role in this field. An exchangeable sequence $\left(Y_{n}\right)$ is a completely symmetric structure in the sense that the distribution of any functional of $\left(Y_{n}\right)$ is the same as that of $\left(Y_{\sigma(n)}\right)$ where $\sigma: \mathbb{N} \rightarrow \mathbb{N}$ is a permutation of the positive integers. Consequently, limit theorems valid for $\left(Y_{n}\right)$ remain valid also for the permuted sequence $\left(Y_{\sigma(n)}\right)$. Hence on the basis of Aldous' theorem it is natural to expect that limit theorems valid for thin subsequences $\left(X_{n_{k}}\right)$ of general tight sequences $\left(X_{n}\right)$ remain valid after any permutation of their terms. This is indeed the case, as proved by Berkes and Tichy [16], but it is far from obvious: the proof of Aldous' theorem is not permutation-invariant and a further thinning of the subsequence constructed in [7] is required to get a subsequence satisfying i.i.d. 
limit theorems after permutations. As far as limit theorems for lacunary trigonometric and related sums under the Hadamard gap condition are concerned, their proofs depend on number-theoretic properties of $\left(n_{k}\right)$ which are, except a few cases, not permutation-invariant and the CLT and LIL for such series can indeed fail after permutations. The purpose of the present chapter is to provide a complete solution of the permutation-invariance of such results. For the proofs we refer to Aistleitner, Berkes and Tichy [2], [3], [5], [6].

Theorem 3.1 Let $\left(n_{k}\right)$ be a sequence of positive integers satisfying (1.1) and let $\sigma: \mathbb{N} \rightarrow \mathbb{N}$ be a permutation of the set of positive integers. Then we have

$$
N^{-1 / 2} \sum_{k=1}^{N} \cos 2 \pi n_{\sigma(k)} x \stackrel{d}{\longrightarrow} N(0,1 / 2)
$$

and

$$
\limsup _{N \rightarrow \infty}(N \log \log N)^{-1 / 2} \sum_{k=1}^{N} \cos 2 \pi n_{\sigma(k)} x=1 \quad \text { a.e. }
$$

where $\stackrel{d}{\longrightarrow}$ denotes convergence in distribution over the probability space $(0,1)$ equipped with the Borel $\sigma$-algebra and the Lebesgue measure.

Note that for the unpermuted CLT and LIL we need much weaker gap conditions than (1.1). In fact, Erdős [26] and Takahashi [48], [49] showed that if a sequence $\left(n_{k}\right)$ of integers satisfies

$$
n_{k+1} / n_{k} \geq 1+k^{-\alpha}, \quad 0 \leq \alpha<1 / 2
$$

then we have the CLT (1.2) and LIL (1.3). Note, however, that (3.3) does not imply permutation-invariance and in fact in Aistleitner, Berkes and Tichy [3] showed that permutation-invariance fails under any gap condition weaker than (1.1).

We pass now to the permutation-invariance of the more general sums $\sum_{k=1}^{n} f\left(n_{k} x\right)$, where $f$ is a measurable function satisfying

$$
f(x+1)=f(x), \quad \int_{0}^{1} f(x) d x=0, \quad \int_{0}^{2} f^{2}(x) d x<\infty
$$

and $\left(n_{k}\right)$ is a sequence of integers satisfying the Hadamard gap condition (1.1). The central limit theorem for $f\left(n_{k} x\right)$ has a long history. Kac [38] proved that if $f$ is Lipschitz continuous then $f\left(n_{k} x\right)$ satisfies the CLT for $n_{k}=2^{k}$ and not much later Erdős and Fortet (see [39], p. 655) showed that this is not any more valid if $n_{k}=2^{k}-1$. Gaposhkin [33] proved that $f\left(n_{k} x\right)$ obeys the CLT if $n_{k+1} / n_{k} \rightarrow \alpha$ where $\alpha^{r}$ is irrational for $r=1,2 \ldots$ and the same holds if all the fractions $n_{k+1} / n_{k}$ are integers. To formulate our results on permutation-invariance, we introduce a series of numbertheoretic conditions playing an important role in the circle of problems studied. We say that a sequence $\left(n_{k}\right)$ of positive integers satisfies

Condition $\mathbf{D}_{2}$, if for any fixed nonzero integers $a, b, c$ the number of solutions of the Diophantine equation

$$
a n_{k}+b n_{l}=c
$$


is bounded by a constant $K(a, b)$, independent of $c$.

Condition $\mathbf{D}_{2}^{(s)}$ (strong $\mathbf{D}_{2}$ ), if for any fixed integers $a \neq 0, b \neq 0, c$ the number of solutions of the Diophantine equation (3.5) is bounded by a constant $K(a, b)$, independent of $c$, where for $c=0$ we require also $k \neq l$.

Condition $\mathbf{D}_{2}^{(w)}$ (weak $\mathbf{D}_{2}$ ), if for any fixed nonzero integers $a, b, c$ the number of solutions of the Diophantine equation

$$
a n_{k}+b n_{l}=c, \quad 1 \leq k, l \leq N
$$

is $o(N)$, uniformly in $c$.

Condition $\mathbf{D}_{2}^{(0)}$, if for any fixed nonzero integers $a, b$ the number of solutions of the Diophantine equation

$$
a n_{k}+b n_{l}=0, \quad 1 \leq k, l \leq N
$$

is $o(N)$.

Condition $\mathbf{D}_{2}$ was introduced by Gaposhkin [33], who proved that under minor smoothness assumptions on $f$, it implies the CLT for $f\left(n_{k} x\right)$. Aistleitner and Berkes [1] proved that the CLT holds for $f\left(n_{k} x\right)$ also under $\mathbf{D}_{2}^{(w)}$ and this condition is necessary. Below we give a precise description of the CLT and LIL behavior of permuted sums $\sum_{k=1}^{N} f\left(n_{\sigma(k)} x\right)$ and in particular, we obtain characterizations of permutation-invariance.

Our first result shows that if we assume the slightly stronger gap condition

$$
n_{k+1} / n_{k} \rightarrow \infty
$$

then the behavior of $f\left(n_{k} x\right)$ is permutation-invariant regardless the number-theoretic structure of $\left(n_{k}\right)$. In what follows, let $\|\cdot\|$ and $\|\cdot\|_{p}$ denote the $L^{2}(0,1)$, resp. $L^{p}(0,1)$ norm.

Theorem 3.2 Let $\left(n_{k}\right)$ be a sequence of positive integers satisfying the gap condition (3.8). Then for any permutation $\sigma: \mathbb{N} \rightarrow \mathbb{N}$ of the integers and for any measurable function $f: \mathbb{R} \rightarrow \mathbb{R}$ satisfying

$$
f(x+1)=f(x), \quad \int_{0}^{1} f(x) d x=0, \quad \operatorname{Var}_{[0,1]} f<+\infty
$$

we have

$$
\frac{1}{\sqrt{N}} \sum_{k=1}^{N} f\left(n_{\sigma(k)} x\right) \stackrel{d}{\longrightarrow} \mathcal{N}\left(0,\|f\|^{2}\right)
$$

and

$$
\limsup _{N \rightarrow \infty} \frac{1}{\sqrt{2 N \log \log N}} \sum_{k=1}^{N} f\left(n_{\sigma(k)} x\right)=\|f\| \quad \text { a.e. }
$$


Assuming only the Hadamard gap condition (1.1), the situation becomes more complex and the number-theoretic structure of $\left(n_{k}\right)$ comes into play. Our next result gives a necessary and sufficient condition for permuted partial sums $\sum_{k=1}^{N} f\left(n_{\sigma(k)} x\right)$ to have only Gaussian limit distributions and gives precise criteria this to happen for a specific permutation $\sigma$.

Theorem 3.3 Let $\left(n_{k}\right)$ be a sequence of positive integers satisfying the Hadamard gap condition (1.1) and condition $\mathbf{D}_{2}$. Let $f$ satisfy (3.9) and let $\sigma$ be a permutation of $\mathbb{N}$. Then $N^{-1 / 2} \sum_{k=1}^{N} f\left(n_{\sigma(k)} x\right)$ has a limit distribution iff

$$
\gamma=\lim _{N \rightarrow \infty} N^{-1} \int_{0}^{1}\left(\sum_{k=1}^{n} f\left(n_{\sigma(k)} x\right)\right)^{2} d x
$$

exists, and then

$$
N^{-1 / 2} \sum_{k=1}^{N} f\left(n_{\sigma(k)} x\right) \stackrel{d}{\longrightarrow} N(0, \gamma) .
$$

Theorem 3.3 is best possible in the following sense:

Theorem 3.4 If condition $\mathbf{D}_{2}$ fails, there exists a permutation $\sigma$ such that the normed partial sums in (3.13) have a nongaussian limit distribution.

In other words, under the Hadamard gap condition and condition $\mathbf{D}_{2}$, the limit distribution of $N^{-1 / 2} \sum_{k=1}^{N} f\left(n_{\sigma(k)} x\right)$ can only be Gaussian, but the variance of the limit distribution depends on the constant $\gamma$ in (3.12) which, as simple examples show, is not permutation-invariant. For example, if $n_{k}=2^{k}$ and $\sigma$ is the identity permutation, then (3.12) holds with

$$
\gamma=\gamma_{f}=\int_{0}^{1} f^{2}(x) d x+2 \sum_{k=1}^{\infty} \int_{0}^{1} f(x) f\left(2^{k} x\right) d x
$$

(see Kac [38]). Using an idea of Fukuyama [29], one can construct permutations $\sigma$ of $\mathbb{N}$ such that

$$
\lim _{N \rightarrow \infty} \frac{1}{N} \int_{0}^{1}\left(\sum_{k=1}^{N} f\left(n_{\sigma(k)} x\right)\right)^{2} d x=\gamma_{\sigma, f}
$$

with $\gamma_{\sigma, f} \neq \gamma_{f}$. Actually, the set of possible values $\gamma_{\sigma, f}$ belonging to all permutations $\sigma$ contains the interval $I_{f}=\left[\gamma_{f},\|f\|^{2}\right]$ and it is equal to this interval provided the Fourier coefficients of $f$ are nonnegative.

Under the slightly stronger condition $\mathbf{D}_{2}^{(s)}$ we get

Theorem 3.5 Let $\left(n_{k}\right)$ be a sequence of positive integers satisfying the Hadamard gap condition (1.1) and condition $\mathbf{D}_{2}^{(s)}$. Let $f$ satisfy (3.9) and let $\sigma$ be a permutation of $\mathbb{N}$. Then the central limit theorem (3.13) holds with $\gamma=\|f\|^{2}$.

We now pass to the problem of the LIL. 
Theorem 3.6 Let $\left(n_{k}\right)$ be a sequence of positive integers satisfying the Hadamard gap condition (1.1) and condition $\mathbf{D}_{2}$. Let $f$ be a measurable function satisfying (3.9), let $\sigma$ be a permutation of $\mathbb{N}$ and assume that

$$
\gamma=\lim _{N \rightarrow \infty} N^{-1} \int_{0}^{1}\left(\sum_{k=1}^{N} f\left(n_{\sigma(k)} x\right)\right)^{2} d x
$$

for some $\gamma \geq 0$. Then we have

$$
\limsup _{N \rightarrow \infty} \frac{\sum_{k=1}^{N} f\left(n_{\sigma(k)} x\right)}{\sqrt{2 N \log \log N}}=\gamma^{1 / 2} \quad \text { a.e. }
$$

Theorem 3.7 Let $\left(n_{k}\right)$ be a lacunary sequence of positive integers satisfying condition $\mathbf{D}_{2}^{(s)}$, and let $f$ be a function satisfying (3.9). Then for any permutation $\sigma: \mathbb{N} \rightarrow \mathbb{N}$ we have

$$
\limsup _{N \rightarrow \infty} \frac{\sum_{k=1}^{N} f\left(n_{\sigma(k)} x\right)}{\sqrt{2 N \log \log N}}=\|f\| \quad \text { a.e. }
$$

Remark. If $f$ is a trigonometric polynomial of degree $d$, then in conditions $\mathbf{D}_{2}$ resp. $\mathbf{D}_{2}^{(s)}$ it suffices to have the bound for the number of solutions of (3.5) for coefficients $a, b$ satisfying $|a| \leq d,|b| \leq d$. Applying this with $d=1$ and using the the fact that for a Hadamard lacunary sequence $\left(n_{k}\right)$ and $c \in \mathbb{Z}$ the number of solutions $(k, l)$, $k \neq l$ of

$$
n_{k} \pm n_{l}=c
$$

is bounded by a constant which is independent of $c$ (see Zygmund [53, p. 203]), we get Theorem 3.1.

All the results formulated so far assume the Hadamard gap condition (1.1) or the stronger condition (3.8). If we drop (1.1), i.e. we allow subexponential sequences $\left(n_{k}\right)$, we need much stronger Diophantine conditions even for the unpermuted CLT and LIL for $f\left(n_{k} x\right)$. Specifically, we need uniform bounds for the number of solutions of Diophantine equations of the type

$$
a_{1} n_{k_{1}}+\ldots+a_{p} n_{k_{p}}=b .
$$

Call a solution of (3.18) nondegenerate if no subsum of the left hand side equals 0 . Let us say that a sequence $\left(n_{k}\right)$ of positive integers satisfies

Condition $\mathbf{A}_{p}$, if there exists a constant $C_{p} \geq 1$ such that for any integer $b \neq 0$ and any nonzero integers $a_{1}, \ldots, a_{p}$ the number of nondegenerate solutions of the Diophantine equation (3.18) is at most $C_{p}$.

The following results are the analogues of the previous results without growth conditions on $\left(n_{k}\right)$.

Theorem 3.8 Let $\left(n_{k}\right)$ be an increasing sequence of positive integers satisfying condition $\mathbf{A}_{p}$ for all $p \geq 2$. Let $f$ satisfy (3.9), let $\sigma$ be a permutation of $\mathbb{N}$ and assume that the limit (3.12) exists. Then the permuted CLT (3.13) is valid. 
Theorem 3.9 Let $\left(n_{k}\right)$ be an increasing sequence of positive integers satisfying condition $\mathbf{A}_{p}$ for all $p \geq 2$ with $C_{p} \leq \exp \left(C p^{\alpha}\right)$ for some $\alpha>0$. Assume also that $f$ satisfies (3.9). Then for any permutation $\sigma$ of $\mathbb{N}$ we have

$$
\limsup _{N \rightarrow \infty} \frac{1}{\sqrt{2 N \log \log N}} \sum_{k=1}^{N} f\left(n_{\sigma(k)} x\right)=\gamma^{1 / 2} \quad \text { a.e. }
$$

Note that for the validity of the LIL we require a specific bound for the constants $C_{p}$ in condition $\mathbf{A}_{p}$. For subexponentially growing $\left(n_{k}\right)$, verifying property $\mathbf{A}_{p}$ is a difficult number-theoretic problem. Classical examples of such sequences are the Hardy-Littlewood-Pólya sequences, i.e. increasing sequences $\left(n_{k}\right)$ consisting of all positive integers of the form $q_{1}^{\alpha_{1}} \cdots q_{\tau}^{\alpha_{\tau}}\left(\alpha_{1}, \ldots \alpha_{\tau} \geq 0\right)$, where $q_{1}, \ldots, q_{\tau}$ is a fixed set of coprime integers. Clearly, such sequences grow subexponentially; Tijdeman [50] proved that

$$
n_{k+1}-n_{k} \geq \frac{n_{k}}{\left(\log n_{k}\right)^{\alpha}}
$$

for some $\alpha>0$, i.e. the growth of $\left(n_{k}\right)$ is almost exponential. Hardy-LittlewoodPólya sequences have remarkable probabilistic and ergodic properties. Nair [44] proved that if $f$ is 1-periodic and integrable in $(0,1)$, then

$$
\lim _{N \rightarrow \infty} \frac{1}{N} \sum_{k=1}^{N} f\left(n_{k} x\right)=\int_{0}^{1} f(t) d t \quad \text { a.e. }
$$

Fukuyama and Petit [30] also showed that the central limit theorem

$$
N^{-1 / 2} \sum_{k=1}^{N} f\left(n_{k} x\right) \stackrel{d}{\longrightarrow} N\left(0, \gamma_{f}^{*}\right)
$$

holds with

$$
\gamma_{f}^{*}=\sum_{k, l:\left(n_{k}, n_{l}\right)=1} \int_{0}^{1} f\left(n_{k} x\right) f\left(n_{l} x\right) d x .
$$

The Diophantine properties of $\left(n_{k}\right)$ have been studied in great detail in recent years. Amoroso and Viada [9] showed recently that Hardy-Littlewood-Pólya sequences satisfy condition $\mathbf{A}_{p}$ for any $p \geq 2$ with $C_{p}=\exp \left(p^{6}\right)$. This is a deep result, involving a substantial sharpening of the subspace theorem of Evertse, Schlickewei and Schmidt (see [28]). Again, the limit $\gamma$ in (3.12) depends on the permutation $\sigma$; one can show that if the Fourier coefficients of $f$ are nonnegative, the class of numbers $\gamma$ contains the interval $\left[\|f\|^{2}, \gamma_{f}^{*}\right]$, with $\gamma_{f}^{*}$ in (3.21).

Since verifying condition $\mathbf{A}_{p}$ for a concrete subexponential sequence $\left(n_{k}\right)$ is difficult, it is worth looking for Diophantine conditions which are strong enough to imply the permutation-invariant CLT and LIL, but which hold for a sufficiently large class of subexponential sequences. In the sequel we will give a such a Diophantine condition $\boldsymbol{A}_{\boldsymbol{\omega}}$. Actually, one can show that this $\boldsymbol{A}_{\boldsymbol{\omega}}$ is satisfied, in a certain statistical sense, for "almost all" sequences $\left(n_{k}\right)$ and thus the permutation-invariant CLT and 
LIL are the "typical" behavior of sequences $f\left(n_{k} x\right)$. Given a nondecreasing sequence $\boldsymbol{\omega}=\left(\omega_{1}, \omega_{2}, \ldots\right)$ of positive integers tending to $+\infty$, let us say that an increasing sequence $\left(n_{k}\right)$ of positive integers satisfies

Condition $\boldsymbol{A}_{\boldsymbol{\omega}}$, if the Diophantine equation

$$
a_{1} n_{k_{1}}+\ldots+a_{p} n_{k_{p}}=0, \quad k_{1}<\ldots<k_{p}, p=\omega_{N},\left|a_{1}\right|, \ldots,\left|a_{p}\right| \leq N^{\omega_{N}}
$$

has no solutions with $k_{p}>N$.

This condition is more technical than $\mathbf{A}_{p}$ and for each $N \geq 1$ it requires a bound for the number of solutions of the $p$-term Diophantine equation $a_{1} n_{k_{1}}+\ldots+a_{p} n_{k_{p}}=0$, where $p=\omega_{N}$ and the coefficients are bounded by $N^{\omega_{N}}$, where we usually choose $\left(\omega_{N}\right)$ to grow slowly.

Theorem 3.10 Let $\boldsymbol{\omega}=\left(\omega_{1}, \omega_{2}, \ldots\right)$ be a nondecreasing sequence of positive integers tending to $+\infty$ and $\left(n_{k}\right)$ a sequence of positive integers satisfying condition $\boldsymbol{A}_{\boldsymbol{\omega}}$. Then for any $f$ satisfying (3.9) and any permutation $\sigma$ of $\mathbb{N}$, relations (3.10) and (3.11) are valid.

Fix $\omega_{N} \rightarrow \infty$. One can show that, in a certain statistical sense, "almost all" sequences $n_{k} \leq k^{\omega_{k}}$ satisfy condition $\boldsymbol{A}_{\boldsymbol{\omega}}$. To make this precise, we need to define a probability measure over the set of such sequences, or, equivalently, a natural random procedure to generate such sequences. Clearly, the simplest procedure is to choose $n_{k}$ independently and uniformly from the integers in the interval $I_{k}=\left[1, k^{\omega_{k}}\right]$ $(k=1,2, \ldots)$. Denote the so obtained measure by $\mu$.

Theorem 3.11 With probability one with respect to $\mu$, the sequence $\left(n_{k}\right)$ satisfies (3.10), (3.11).

Clearly, for slowly increasing $\left(\omega_{k}\right)$ the so obtained sequence grows almost polynomially (as a comparison, Hardy-Littlewood-Pólya sequences grow almost exponentially by (3.20)). We do not know if there exist polynomially growing sequences $\left(n_{k}\right)$ satisfying any of (3.10), (3.11). As a simple combinatorial argument shows, sequences $\left(n_{k}\right)$ satisfying $\mathbf{A}_{p}$ for all $p \geq 2$ cannot grow polynomially.

\section{Lacunary sequences outside $L^{2}$}

For lacunary series $\sum c_{k} \varphi_{n_{k}}$ in $L^{2}$, a number of interesting uniform limit theorems have been obtained by ad hoc methods, such as e.g., the selection theorem of Menshov [42] or its permutation-invariant version by Komlós [40], already mentioned in the Introduction. A further example is the result of Gaposhkin [32] stating that for every uniformly bounded sequence $\left(X_{n}\right)$ of random variables there exists a subsequence $\left(X_{n_{k}}\right)$ and random variables $X$ and $Y \geq 0$ such that if $\left(a_{k}\right)$ is a sequence of real numbers satisfying

$$
a_{N}=o\left(A_{N}\right) \quad \text { with } \quad A_{N}=\left(\sum_{k=1}^{N} a_{k}^{2}\right)^{1 / 2},
$$


then

$$
A_{N}^{-1} \sum_{k=1}^{N} a_{k}\left(X_{n_{k}}-X\right) \stackrel{d}{\longrightarrow} N(0, Y)
$$

and if

$$
a_{N}=o\left(A_{N} /\left(\log \log A_{N}\right)^{1 / 2}\right)
$$

then

$$
\limsup _{N \rightarrow \infty}\left(2 A_{N}^{2} \log \log A_{N}\right)^{-1 / 2} \sum_{k=1}^{N} a_{k}\left(X_{n_{k}}-X\right)=Y^{1 / 2} \quad \text { a.s. }
$$

Note that the conditions (4.1) and (4.3) are the classical (and optimal) conditions for a weighted sum $\sum_{k=1}^{N} a_{k} X_{k}$ of bounded independent random variables with mean 0 to satisfy the central limit theorem and law of the iterated logarithm. In contrast, no uniform results seem to be known for sequences $\left(X_{n}\right)$ which are not in $L^{2}$ and the purpose of this section is to formulate such results.

Our first result is the analogue of the uniform CLT (4.1)-(4.2) in the case when the limit distribution is, instead of the Gaussian law, a symmetric stable distribution $G_{\alpha, c}$ with parameter $0<\alpha<2$, having characteristic function (Fourier transform) $\exp \left(-c|t|^{\alpha}\right)$. By a classical result in probability theory, if $0<\alpha<2,\left(X_{n}\right)$ is a sequence of i.i.d. symmetric random variables with characteristic function $\varphi$ satisfying

$$
\varphi(t)=1-c|t|^{\alpha}+o\left(|t|^{\alpha}\right) \quad \text { as } t \rightarrow 0
$$

and $\left(a_{k}\right)$ is a numerical sequence with

$$
\max _{1 \leq k \leq N}\left|a_{k}\right|=o\left(A_{N}\right), \quad A_{N}=\left(\sum_{k=1}^{N}\left|a_{k}\right|^{\alpha}\right)^{1 / \alpha},
$$

then

$$
A_{N}^{-1 / \alpha} \sum_{k=1}^{N} a_{k} X_{k} \stackrel{d}{\longrightarrow} G_{\alpha, c} .
$$

Using Theorem 2.1, Berkes and Tichy [17] proved the corresponding uniform limit theorem for lacunary series.

Theorem 4.1 Let $0<\alpha<2, c>0$ and let $\left(X_{n}\right)$ be a determining sequence of r.v.'s with limit random measure $\mu$. Assume that the characteristic function $\varphi$ of $\mu$ satifies (4.5) with probability 1 . Then there exists a subsequence $\left(X_{n_{k}}\right)$ such that for any real sequence $\left(a_{k}\right)$ satisfying (4.6) we have

$$
A_{N}^{-1} \sum_{k=1}^{N} a_{k} X_{n_{k}} \stackrel{d}{\longrightarrow} G_{\alpha, c}
$$

Using the same method, one can prove analogous uniform limit theorems for partial maxima, sample extrema and other nonlinear functionals of lacunary series without assuming finite second moments, but instead of elaborating on this, we investigate lacunary subsequences $f\left(n_{k} x\right)$ of the dilated system $f(n x)$, already studied 
in the previous section, and formulate very general uniform results for their lacunary subsequences $f\left(n_{k} x\right)$ without assuming the square integrability of (or, for that matter, any integrability condition for) the periodic function $f$. Our starting point is Gaposhkin's observation [34] that sufficiently thin subsequences of $f(n x)$ are almost independent in a very strong sense.

Theorem 4.2 Let $f: \mathbb{R} \rightarrow \mathbb{R}$ be a measurable function with period 1. Then there exists an increasing sequence $\left(n_{k}\right)$ of positive integers and measurable functions $g_{k}(x)$, $k=1,2, \ldots$ on $(0,1)$ such that the $g_{k}$ are stochastically independent and

$$
f\left(n_{k} x\right)=g_{k}(x)+\psi_{k}(x)+\eta_{k}(x)
$$

where

$$
\sum_{k=1}^{\infty}\left\|\psi_{k}\right\|_{M}<\infty \quad \text { and } \quad \sum_{k=1}^{\infty} \mu\left\{x: \eta_{k}(x) \neq 0\right\}<\infty .
$$

Here $\|\cdot\|_{M}$ denotes the norm in the space $M(0,1)$ of measurable functions on $(0,1)$ defined by $\|\psi\|_{M}=\inf \{\epsilon>0: \mu(x:|\psi(x)| \geq \epsilon) \leq \epsilon\}$. If $f \in L^{p}(0,1)(p \geq 1)$ or $f \in$ $C(0,1)$, then the conclusion remains valid with the $g_{k}$ belonging to the corresponding spaces and $\|\cdot\|_{M}$ replaced by $\|\cdot\|_{p}$ or $\|\cdot\|_{C}$, respectively.

As an immediate consequence, we get

$$
\sum_{k=1}^{\infty}\left|f\left(n_{k} x\right)-g_{k}(x)\right|<\infty \quad \text { a.e. }
$$

in all cases covered by the theorem. Clearly (4.9) extends any limit theorem $T$ from $\left(g_{k}\right)$ to $\left(f\left(n_{k} x\right)\right)$ which has the property

$$
\left(X_{k}\right) \text { satisfies } T \text { and } \sum_{k=1}^{\infty}\left|X_{k}-X_{k}^{\prime}\right|<\infty \text { a.s. } \Longrightarrow\left(X_{k}^{\prime}\right) \text { satisfies } T \text {. }
$$

Moreover, relation (4.9) remains valid after any permutation of the indices and thus limit theorems for $f\left(n_{k} x\right)$ provided by Gaposhkin's theorem are automatically permutation-invariant. We observe further that by (4.9) we have $f\left(n_{k} x\right)-g_{k}(x) \rightarrow 0$ a.s. and thus given any sequence $\Psi(k) \rightarrow \infty$ one can select a subsequence $\left(m_{k}\right)$ such that

$$
\sum_{k=1}^{\infty} \Psi(k)\left|f\left(n_{m_{k}} x\right)-g_{m_{k}}(x)\right|<\infty \quad \text { a.e. }
$$

Thus, if $\left(a_{k}\right)$ is a weight sequence such that $\left|a_{k}\right| \leq \Psi(k)$, then

$$
\left|\sum_{k=1}^{N} a_{k} f\left(n_{m_{k}} x\right)-\sum_{k=1}^{N} a_{k} g_{m_{k}}(x)\right|=O(1) \quad \text { a.e. }
$$

a relation leading to uniform limit theorems of weighted sums of $f\left(n_{m_{k}} x\right)$ by using the corresponding limit theorem for weighted sums of independent random variables. The only shortcoming of Gaposhkin's result that it gives no information on the growth 
speed of $\left(n_{k}\right)$ : the construction of $\left(n_{k}\right)$, although theoretically explicit, is very complicated and yields an extremely rapidly growing $\left(n_{k}\right)$. Since all classical lacunarity results for "concrete" function systems involve concrete lacunarity conditions (such as the Hadamard gap condition), it would be desirable to give the dependence of the growth of $\left(n_{k}\right)$ in Gaposhkin's theorem on the function $f$ explicitly. In the sequel, we formulate such explicit results; for the proofs see Aistleitner, Berkes and Tichy [4].

Theorem 4.3 Let $\left(n_{k}\right)$ be an increasing sequence of positive integers. Then there exists a sequence $\left(g_{k}\right)$ of measurable functions on $(0,1)$, independent and uniformly distributed over $(0,1)$ in the sense of probability theory, such that

$$
\lambda\left\{x \in(0,1):\left|\left\langle n_{k} x\right\rangle-g_{k}(x)\right| \geq 2 n_{k} / n_{k+1}\right\} \leq 2 n_{k} / n_{k+1} \quad k=1,2, \ldots
$$

where $\langle\cdot\rangle$ denotes fractional part.

Note that Theorem 4.3 concerns the specific sequence $\left\langle n_{k} x\right\rangle$, but depending on the properties of the function $f$, it leads automatically to a corresponding approximation theorem for general sequences $\left(f\left(n_{k} x\right)\right)$. For example, if $f$ is continuous with continuity modulus $\omega(f, \delta)$, then (4.11) implies

$$
\sum_{k=1}^{\infty}\left|f\left(n_{k} x\right)-g_{k}^{*}(x)\right|<\infty \quad \text { a.e. }
$$

with $g_{k}^{*}(x)=f\left(g_{k}(x)\right)$, provided

$$
\sum_{k=1}^{\infty} n_{k} / n_{k+1}<\infty, \quad \sum_{k=1}^{\infty} \omega\left(f, n_{k} / n_{k+1}\right)<\infty
$$

A much more general consequence of Theorem 4.3 is the following

Corollary 4.1 Let $f: \mathbb{R} \rightarrow \mathbb{R}$ be a measurable function with period 1 and $\left(n_{k}\right)$ an increasing sequence of positive integers. Let $T_{k}$ be positive numbers such that

$$
\lambda\left\{f \in(0,1):|f| \geq T_{k}\right\} \leq k^{-2} \quad(k=1,2, \ldots)
$$

and assume that

$$
\sum_{k=1}^{\infty}\left[\left(n_{k} / n_{k+1}\right)^{1 / 4} T_{k}+\omega_{2}\left(f_{T_{k}},\left(n_{k} / n_{k+1}\right)^{1 / 4}\right)\right]<\infty .
$$

Then there exists a sequence $\left(g_{k}^{*}\right)$ of measurable functions on $(0,1)$, independent and having the same distribution over $(0,1)$ as $f$, such that (4.12) holds.

Here

$$
\omega_{2}(f, \delta)=\sup _{0 \leq h \leq \delta}\left(\int_{0}^{1}|f(x+h)-f(x)|^{2} d x\right)^{1 / 2}
$$


is the $L^{2}$ modulus of continuity of $f$ and $f_{T}$ denotes the truncated function $f \cdot I\{|f| \leq$ $T\}$.

Given any periodic measurable function $f$, one can choose $T_{k}$ so that (4.13) holds, and then (4.14) becomes a "concrete" gap condition. Clearly, the 'larger' the function $f$ is, the faster $n_{k}$ tends to $\infty$. In particular for functions $f$ not belonging to any $L^{p}$, $p>0,\left(n_{k}\right)$ is growing very fast. We illustrate the procedure on two classical limit theorems mentioned above.

Corollary 4.2 Let $f: \mathbb{R} \rightarrow \mathbb{R}$ be a measurable function with period 1 such that the distribution function

$$
F(x)=\mu\{t \in(0,1): f(t) \leq x\}
$$

satisfies

$$
1-F(x) \sim p x^{-\alpha} L(x), \quad F(-x) \sim q x^{-\alpha} L(x) \quad \text { as } x \rightarrow \infty
$$

for some constants $p, q \geq 0, p+q=1,0<\alpha<2$ and a slowly varying function $L$. Let $\left(n_{k}\right)$ be an increasing sequence of positive integers satisfying (4.14). Then letting $S_{n}=\sum_{k=1}^{n} f\left(n_{k} x\right)$, we have

$$
\left(S_{n}-a_{n}\right) / b_{n} \stackrel{d}{\longrightarrow} G_{\alpha}
$$

for some numerical sequences $\left(a_{n}\right),\left(b_{n}\right)$ and an $\alpha$-stable distribution $G_{\alpha}$.

Corollary 4.3 Let $f: \mathbb{R} \rightarrow \mathbb{R}$ be a measurable function with period 1 such that the distribution function $F$ of $f$ in (4.15) satisfies $F(x)<1$ for all $x$ and $1-F$ is regularly varying at $+\infty$ in the Karamata sense with a negative exponent $-\alpha$. Let $\left(n_{k}\right)$ be an increasing sequence of positive integers satisfying (4.14). Then letting $M_{n}=\max _{1 \leq k \leq n} f\left(n_{k} x\right)$, we have

$$
\left(M_{n}-a_{n}\right) / b_{n} \stackrel{d}{\longrightarrow} H_{\alpha}
$$

where $H_{\alpha}(x)=\exp \left(-x^{-\alpha}\right) I_{(0, \infty)}(x)$.

Note that (4.16) is the classical necessary and sufficient condition for the partial sums $S_{n}$ of an i.i.d. sequence with distribution function $F$ to satisfy the limit theorem (4.17) with suitable norming and centering sequences $\left(a_{n}\right),\left(b_{n}\right)$. Corollary 4.2 shows that if the distribution function $F$ of the periodic function $f$ satisfies (4.16), then the partial sums of $f\left(n_{k} x\right)$ for any $\left(n_{k}\right)$ satisfying (4.14) obey the stable limit theorem (4.17). Similarly, the assumption on $F$ in Corollary 4.3 is the well known necessary and sufficient condition for the centered and normed maxima of an i.i.d. sequence with distribution $F$ to converge weakly to the distribution $H(x)=\exp \left(-x^{-\alpha}\right) I_{(0, \infty)}(x)$, the so called Fréchet distribution. As we know (see e.g. [31]), the limit distribution in (4.18) for any i.i.d. sequence can be only one of the Fréchet, Gumbel and Weibull distributions with respective distribution functions $\exp \left(-x^{-\alpha}\right) I_{(0, \infty)}(x), \exp \left(-(-x)^{\alpha}\right)\left(1-I_{(0, \infty)}(x)\right)$ and $\exp \left(-e^{-x}\right)$. The analogue of Corollary 4.3 holds for the other two limiting classes, too. 


\section{Applications to Banach space theory}

It is an old (and only partially solved) problem of Banach space theory is to characterize Banach spaces containing an isomorphic copy of classical spaces like $L^{p}(0,1)$, $C(0,1)$, etc. A modern approach to this problem via lacunary series is due to Kadec and Pełczyński [37]. Call two sequences $\left(x_{n}\right)$ and $\left(y_{n}\right)$ in a Banach space $(B,\|\cdot\|)$ equivalent if there exists a constant $K>0$ such that

$$
K^{-1}\left\|\sum_{i=1}^{n} a_{i} x_{i}\right\| \leq\left\|\sum_{i=1}^{n} a_{i} y_{i}\right\| \leq K\left\|\sum_{i=1}^{n} a_{i} x_{i}\right\|
$$

for every $n \geq 1$ and every $\left(a_{1}, \ldots, a_{n}\right) \in \mathbb{R}^{n}$. Clearly, in this case the subspaces of $B$ spanned by $\left(x_{n}\right)$ and $\left(y_{n}\right)$ are isomorphic. In particular, if $\left(x_{n}\right)$ is a sequence in $L^{p}(0,1), p \geq 1$ such that for some constant $K>0$

$$
K^{-1}\left(\sum_{k=1}^{N} a_{k}^{2}\right)^{1 / 2} \leq\left\|\sum_{k=1}^{N} a_{k} x_{k}\right\|_{p} \leq K\left(\sum_{k=1}^{N} a_{k}^{2}\right)^{1 / 2}
$$

or

$$
K^{-1}\left(\sum_{k=1}^{N}\left|a_{k}\right|^{p}\right)^{1 / p} \leq\left\|\sum_{k=1}^{N} a_{k} x_{k}\right\|_{p} \leq K\left(\sum_{k=1}^{N}\left|a_{k}\right|^{p}\right)^{1 / p}
$$

then the subspace spanned by $\left(x_{n}\right)$ is isomorphic to $\ell^{2}$ and $\ell^{p}$, respecively. Kadec and Pelczynski [37] proved that for any normalized weakly null sequence $\left(x_{n}\right)$ in $L^{p}(0,1), p>2$ there exists a subsequence $\left(x_{n_{k}}\right)$ which is equivalent to the unit vector basis of $\ell^{2}$ or $\ell^{p}$. Thus every infinite dimensional closed subspace $H$ of $L^{p}(0,1)$, $p>2$ contains a subspace isomorphic to $\ell^{2}$ or $\ell^{p}$. In the case when $\left(x_{n}\right)$ is uniformly integrable in $L^{p}(0,1)$ then the first alternative holds, while if the functions $\left(x_{n}\right)$ have disjoint support, the second alternative holds trivially with $\varepsilon=0$. The general case follows via a subsequence splitting argument as in [37].

In the case of a sequence $\left(x_{n}\right) \in L^{p}, 1 \leq p<2$ the problem is considerably harder. Sufficient conditions for the existence of a subsequence equivalent to the unit vector basis of $\ell^{2}$ were given by Berkes [11] and Guerre [36]. The purpose of the present paper is to give a complete solution of the problem. Namely, Berkes and Tichy [18] proved the following

Theorem 5.1 Let $1 \leq p<2$ and let $\left(X_{n}\right)$ be a determining sequence of random variables such that $\left\|X_{n}\right\|_{p}=1(n=1,2, \ldots),\left\{\left|X_{n}\right|^{p}, n \geq 1\right\}$ is uniformly integrable and $X_{n} \rightarrow 0$ weakly in $L^{p}{ }^{1}$ Let $\mu$ be the limit random measure of $\left(X_{n}\right)$. Then there exists a subsequence $\left(X_{n_{k}}\right)$ equivalent to the unit vector basis of $\ell^{2}$ if and only if

$$
\int_{-\infty}^{\infty} x^{2} d \mu(x) \in L^{p / 2}
$$

\footnotetext{
${ }^{1}$ This is meant as $\lim _{n \rightarrow \infty} \mathbb{E}\left(X_{n} Y\right)=0$ for all $Y \in L_{q}$ where $1 / p+1 / q=1$. To avoid confusion with weak convergence of probability distributions, the latter will be called convergence in distribution.
} 
Note that (5.1), (5.2) are required for all $\left(a_{1}, \ldots, a_{n}\right) \in \mathbb{R}^{n}$ and all $n \geq 1$ with the same constant $K$, i.e. they are uniform statements. Using an observation of Aldous [7], such a uniformity can be obtained from an equicontinuity statement. Let $\left(X_{n}\right)$ be a determining sequence in $L^{p}(0,1)$ with limit random measure $\mu$ and limit exchangeable sequence $\left(Y_{n}\right)$. Our purpose is to construct, given $\varepsilon>0$, a sequence $n_{1}<n_{2}<\cdots$ of integers such that

$$
(1-\varepsilon) \psi\left(a_{1}, \ldots, a_{k}\right) \leq\left\|\sum_{i=1}^{k} a_{i} X_{n_{i}}\right\|_{p} \leq(1+\varepsilon) \psi\left(a_{1}, \ldots, a_{k}\right)
$$

for every $k \geq 1$ and $\left(a_{1}, \ldots, a_{k}\right) \in \mathbb{R}^{k}$ where

$$
\psi\left(a_{1}, \ldots, a_{n}\right)=\left\|\sum_{i=1}^{n} a_{i} Y_{i}\right\|_{p} .
$$

To construct $n_{1}$ we set

$$
\begin{aligned}
Q(\mathbf{a}, n, \ell) & =\left|a_{1} X_{n}+a_{2} Y_{2}+\cdots+a_{\ell} Y_{\ell}\right|^{p} \\
R(\mathbf{a}, \ell) & =\left|a_{1} Y_{1}+a_{2} Y_{2}+\cdots+a_{\ell} Y_{\ell}\right|^{p}
\end{aligned}
$$

for every $n \geq 1, \ell \geq 2$ and $\mathbf{a}=\left(a_{1}, \ldots, a_{\ell}\right) \in \mathbb{R}^{\ell}$. We claim that

$$
E\left\{\frac{Q(\mathbf{a}, n, \ell)}{\psi(\mathbf{a})^{p}}\right\} \longrightarrow E\left\{\frac{R(\mathbf{a}, \ell)}{\psi(\mathbf{a})^{p}}\right\} \text { as } n \rightarrow \infty \text { uniformly in a, } \ell
$$

(The right side of (5.4) equals 1.) To do this we note that by the properties of the limit exchangeable sequence, relation (5.4) holds for every fixed vector $\mathbf{a}=\left(a_{1}, \ldots, a_{\ell}\right)$ and by a well known result of Ranga Rao [46], for uniformity we have to verify a certain equicontinuity property of the functions $Q(\mathbf{a}, n, \ell) / \psi(\mathbf{a})^{p}$ in (5.4). If $n_{1}<n_{2}<\ldots<$ $n_{k-1}$ are already constructed, an analogous equicontinuity argument implies that for $\ell>k$

$$
\begin{aligned}
& \psi(\mathbf{a})^{-1}\left\|a_{1} X_{n_{1}}+\cdots+a_{k-1} X_{n_{k-1}}+a_{k} X_{n}+a_{k+1} Y_{k+1}+\cdots+a_{\ell} Y_{\ell}\right\|_{p} \\
& \longrightarrow \psi(\mathbf{a})^{-1}\left\|a_{1} X_{n_{1}}+\cdots+a_{k-1} X_{n_{k-1}}+a_{k} Y_{k}+\cdots+a_{\ell} Y_{\ell}\right\|_{p} \text { as } n \rightarrow \infty
\end{aligned}
$$

uniformly in a and $\ell$, a relation that can be used to choose $n_{k}$. Carrying out this argument, we get the following general theorem, proved in Berkes [11]. Let $\mathcal{M}$ be the set of all probability measures on $\mathbb{R}^{1}$ and let $\pi$ be the Prohorov metric on $\mathcal{M}$ defined by

$$
\begin{aligned}
& \pi(\nu, \lambda)=\inf \left\{\varepsilon>0: \nu(A) \leq \lambda\left(A^{\varepsilon}\right)+\varepsilon\right. \text { and } \\
& \left.\quad \lambda(A) \leq \nu\left(A^{\varepsilon}\right)+\varepsilon \text { for all Borel sets } A \subset \mathbb{R}^{1}\right\} .
\end{aligned}
$$

Here $A^{\varepsilon}$ denotes the open $\varepsilon$-neighbourhood of $A$. 
Theorem 5.2 Let $p \geq 1$ and let $\left(X_{n}\right)$ be a sequence of r.v.'s so that $\left\{\left|X_{n}\right|^{p}, n \geq 1\right\}$ is uniformly integrable. Let $\mu$ and $\left(Y_{n}\right)$ denote the limit random measure and limit exchangeable sequence of $\left(X_{n}\right)$, respectively. Let $S$ be a Borel subset of $(\mathcal{M}, \pi)$ such that $\mu$ is concentrated on $S$ with probability 1. Assume that there exists a separable metric d on $S$, Borel-equivalent to the Prohorov metric $\pi$ such that $\mathbb{E} d(\mu, 0)^{p}<+\infty$ (0 denotes the zero distribution) and

$$
\left|\frac{\left\|t+\sum_{k=1}^{n} a_{k} \xi_{k}^{(\nu)}\right\|_{p}-\left\|t+\sum_{k=1}^{n} a_{k} \xi_{k}^{(\lambda)}\right\|_{p}}{\left\|\sum_{k=1}^{n} a_{k} Y_{k}\right\|_{p}}\right| \leq d(\nu, \lambda)
$$

for every $n \geq 1, \nu, \lambda \in S$, real numbers $t, a_{1}, \ldots, a_{n}$ and i.i.d. sequences $\left(\xi_{n}^{(\nu)}\right),\left(\xi_{n}^{(\lambda)}\right)$ with respective distributions $\nu$ and $\lambda$. Then for every $\varepsilon>0$ there exists an increasing sequence $\left(n_{k}\right)$ of positive integers such that

$$
(1-\varepsilon) \psi\left(a_{1}, \ldots, a_{k}\right) \leq\left\|\sum_{i=1}^{k} a_{i} X_{n_{i}}\right\|_{p} \leq(1+\varepsilon) \psi\left(a_{1}, \ldots, a_{k}\right)
$$

for every $k \geq 1$ and $\left(a_{1}, \ldots, a_{k}\right) \in \mathbb{R}^{k}$.

Condition (5.5) is the crucial equicontinuity assumption assuring uniformity in (5.6). The proof of Theorem 5.1 uses Theorem 5.2, Theorem 2.1 and concentration arguments.

Theorem 5.2 remains valid if we replace the linear functional $\left\|\sum_{k=1}^{n} a_{k} x_{k}\right\|$ by a general functional $f_{n}\left(a_{1} x_{1}, \ldots, a_{n} x_{n}\right)$; the only difference is that the equicontinuty condition (5.5) should be replaced by the corresponding assumption for the functional $f_{n}$ :

Theorem 5.3 Let $\left(X_{n}\right)$ be a tight sequence of r.v.'s and $f_{k}: \mathbb{R}^{k} \rightarrow \mathbb{R}(k=1,2, \ldots)$ be measurable functions. Let $\mu$ and $\left(Y_{n}\right)$ denote the limit random measure and limit exhangeable sequence of $\left(X_{n}\right)$, respecively. Put

$$
\psi^{*}\left(a_{1}, \ldots, a_{k}\right)=\mathbb{E} f_{k}\left(a_{1} Y_{1}, \ldots, a_{k} Y_{k}\right) .
$$

Let $S$ be a Borel subset of $(\mathcal{M}, \pi)$ such that $P(\mu \in S)=1$. Assume that the following conditions are satisfied:

(a) $\left|\psi^{*}\left(a_{1}, \ldots, a_{k}\right)\right| \geq\left|\psi^{*}\left(a_{1}, 0, \ldots, 0\right)\right|$,

(b) There exists a separable metric d on $S$, Borel-equivalent to the Prohorov metric $\pi$ such that

$$
\begin{gathered}
\left|\mathbb{E} f_{k+1}\left(t, a_{1} \xi_{1}^{(\nu)}, \ldots, a_{k} \xi_{k}^{(\nu)}\right)-\mathbb{E} f_{k+1}\left(t^{\prime}, a_{1} \xi_{1}^{(\lambda)}, \ldots, a_{k} \xi_{k}^{(\lambda)}\right)\right| \\
\leq\left|t-t^{\prime}\right|+\psi^{*}\left(a_{1}, \ldots, a_{k}\right) d(\nu, \lambda)
\end{gathered}
$$

for every $k \geq 1, \nu, \lambda \in S$, real numbers $t, a_{1}, \ldots, a_{k}$ and i.i.d. sequences $\left(\xi_{n}^{(\nu)}\right),\left(\xi_{n}^{(\lambda)}\right)$ with respective distributions $\nu$ and $\lambda$.

Then for every $\varepsilon>0$ there exists a subsequence $\left(X_{n_{k}}\right)$ such that

$$
(1-\varepsilon) \psi^{*}\left(a_{1}, \ldots, a_{k}\right) \leq \mathbb{E} f_{k}\left(a_{1} X_{n_{1}}, \ldots, a_{k} X_{n_{k}}\right) \leq(1+\varepsilon) \psi^{*}\left(a_{1}, \ldots, a_{k}\right)
$$

for any $k \geq 1$ and any $\left(a_{1}, \ldots, a_{k}\right) \in \mathbb{R}^{k}$. 
Theorem 5.3 states the uniform asymptotic behavior of $\mathbb{E} f_{k}\left(a_{1} X_{n_{1}}, \ldots, a_{k} f_{n_{k}}\right)$ for lacunary sequences $\left(n_{k}\right)$ and general functionals $f_{k}$. The equicontinuity condition (5.7) can be readily verified for various functionals $f_{k}$ corresponding to actual limit theorems, leading to a widely applicable uniform version of the subsequence principle. For a detailed discussion and for the proof of the theorem we refer to Berkes and Tichy [20].

\section{Resonance theorems}

Call a sequence $\left(f_{n}\right)$ of measurable functions on $(0,1)$ a convergence system in measure if for any real sequence $\left(c_{n}\right)$ with $\sum_{n=1}^{\infty} c_{n}^{2}<\infty$ the series $\sum_{n=1}^{\infty} c_{n} f_{n}$ converges in measure. The following interesting result was proved by Nikishin [45]:

Theorem. A function system $\left(f_{n}\right)$ over $(0,1)$ is a convergence system in measure if and only if for any $\varepsilon>0$ there exists a measurable set $A_{\varepsilon} \subset(0,1)$ with measure exceeding $1-\varepsilon$ and a constant $K_{\varepsilon}>0$ such that for all $N \geq 1,\left(a_{1}, \ldots, a_{N}\right) \in \mathbb{R}^{N}$ we have

$$
\int_{A_{\varepsilon}}\left(\sum_{k=1}^{N} a_{k} f_{k}\right)^{2} d x \leq K_{\varepsilon} \sum_{k=1}^{N} a_{k}^{2} .
$$

The sufficiency of (6.1) is obvious from Cauchy's criterion, the crucial statement is the converse: if a sequence $\left(f_{n}\right)$ is a convergence system in measure then, except a subset of $(0,1)$ with arbitrary small measure, $\left(f_{n}\right)$ behaves like an orthonormal sequence. For reasons explained in [45], p. 128 such a theorem, and its analogue for function series $\sum_{n=1}^{\infty} c_{n} f_{n}$ with $\left(c_{n}\right) \in \ell^{p}, 1 \leq p \leq \infty$ are called resonance theorems. The purpose of the present section is to give analogues of Nikishin's resonance theorem for the central limit theorem. Our first result is

Theorem 6.1 Let $\left(X_{n}\right)$ be a sequence of random variables over a probability space $(\Omega, \mathcal{F}, P)$ such that for any bounded real sequence $\left(a_{n}\right)$ satisfying

$$
A_{N}^{2}:=\sum_{k=1}^{N} a_{k}^{2} \rightarrow \infty
$$

we have

$$
\frac{1}{A_{N}} \sum_{k=1}^{N} a_{k} X_{k} \stackrel{d}{\longrightarrow} N(0,1) .
$$

Then for any $\varepsilon>0$ there exists a set $A \subset \Omega$ with $P(A) \geq 1-\varepsilon$ such that

$$
\sup _{n} \int_{A} X_{n}^{2} d P<\infty .
$$

Note that we do not assume here the independence (or anything about the joint distribution) of the $X_{n}$. Because of that, the converse of the theorem is obviously 
false. As we will see, however, a necessary and sufficient "almost $L^{2}$ type" characterization of the weighted CLT can be given in the lacunary case. Call a sequence $\left(X_{n}\right)$ of r.v.'s nontrivial if it has no subsequence converging with positive probability.

Theorem 6.2 Let $\left(X_{n}\right)$ be a nontrivial sequence of r.v.'s. Then the following statements are equivalent:

(A) There exists a subsequence $\left(X_{n_{k}}\right)$ and r.v.'s $X, Y$ with $Y>0$ such that for all further subsequences $\left(X_{m_{k}}\right)$ of $\left(X_{n_{k}}\right)$ we have

$$
\frac{\sum_{k=1}^{N}\left(X_{m_{k}}-X\right)}{Y \sqrt{N}} \stackrel{d}{\longrightarrow} N(0,1)
$$

relatively to any set $A \subset \Omega$ with $P(A)>0$.

(B) For every $\varepsilon>0$ there is a subsequence $\left(X_{n_{k}}\right)$ and a set $A \subset \Omega$ with $P(A) \geq 1-\varepsilon$ such that

$$
\sup _{k} \int_{A} X_{n_{k}}^{2} d P<+\infty .
$$

If $\left(X_{n}\right)$ is determining with limit random measure $\mu$, a further equivalent statement is:

(C) We have

$$
\int_{-\infty}^{+\infty} x^{2} d \mu(x)<+\infty \quad \text { a.s. }
$$

For the proof we refer to Berkes and Tichy [19].

\section{Series with random gaps}

In this chapter we investigate the behavior of trigonometric sums $S_{N}=\sum_{k=1}^{N} \sin n_{k} x$, where $\left(n_{k}\right)$ is an increasing random sequence of integers. There are many different types of such random sequences and we investigate the simplest case when $n_{1}, n_{2}, \ldots$ are independent random variables having discrete uniform distribution on disjoint blocks $I_{1}, I_{2}, \ldots$ of integers. The case when $\cup_{k=1}^{\infty} I_{k}=\mathbb{N}$ and $\left|I_{k}\right|$ is constant, or tend to $+\infty$ was settled by Berkes [10] and Bobkov and Götze [21] and in the present chapter we investigate the case of general $I_{k}$, exhibiting a number of interesting new phenomena. We show that $S_{N}$ has a decomposition $S_{N}^{(1)}+S_{N}^{(2)}$, where $S_{N}^{(1)}$ satisfies, with probability 1 , a self-normalized central limit theorem and under mild regularity conditions on the sizes $\left|I_{k}\right|$ of the blocks $I_{k}, S_{N}^{(1)} / \sqrt{N}$ has a pure or mixed Gaussian limit distribution. Moreover, $S_{N}^{(2)}$ is a nonrandom trigonometric sum, asymptotically independent of $S_{N}^{(1)}$, whose asymptotic distribution depends sensitively of the gaps $\Delta_{k}$ between the blocks $I_{k}$ and which can be nongaussian. 
Theorem 7.1 Let $I_{1}, I_{2}, \ldots$ be disjoint intervals of positive integers with cardinalities $\left|I_{k}\right| \geq 2$ and let $n_{1}, n_{2}, \ldots$ be independent random variables defined on a probability space $(\Omega, \mathcal{A}, \mathbb{P})$ such that $n_{k}$ is uniformly distributed on $I_{k}$. Let

$$
\lambda_{k}(x)=\mathbb{E}\left(\sin n_{k} x\right), \quad \gamma_{N}^{2}(x)=\sum_{k=1}^{N}\left(\sin n_{k} x-\lambda_{k}(x)\right)^{2} .
$$

Then $\mathbb{P}$-almost surely

$$
\frac{1}{\gamma_{N}(x)} \sum_{k=1}^{N}\left(\sin n_{k} x-\lambda_{k}(x)\right) \stackrel{d}{\longrightarrow} N(0,1)
$$

with respect to the probability space $((0,2 \pi), \mathcal{B}, \lambda)$, where $\mathcal{B}$ is the Borel $\sigma$-algebra and $\lambda$ is normalized Lebesgue measure on $(0,2 \pi)$. If the asymptotic densities $\mu_{d}$ of the sets $\left\{k \in \mathbb{N}:\left|I_{k}\right|=d\right\}, d=1,2, \ldots$ exist, then for every $x \in \mathbb{R}$ we have

$$
\lim _{N \rightarrow \infty} \frac{\gamma_{N}^{2}(x)}{N}=g(x) \quad \mathbb{P}-\text { a.s. }
$$

where

$$
g(x)=\frac{1}{2}-\sum_{d=1}^{\infty} \frac{\sin ^{2}(d x / 2)}{d^{2} \sin ^{2}(x / 2)} \mu_{d}
$$

and

$$
\frac{1}{\sqrt{N}} \sum_{k=1}^{N}\left(\sin n_{k} x-\lambda_{k}(x)\right) \stackrel{d}{\longrightarrow} N(0, g) .
$$

Note that the self-normalized CLT (7.2) holds for $\sin n_{k} x-\lambda_{k}(x)$ without any regularity condition on the sequence $\left|I_{k}\right|$; in particular, the existence of the asymptotic densities $\mu_{d}$ is not required for (7.5). Without the existence of $\mu_{d}$, however, the sequence $\gamma_{N}(x)^{2} / N$ in (7.3) can converge to different functions $g$ along different subsequences and thus the limit distribution of $N^{-1 / 2} \sum_{k=1}^{N}\left(\sin n_{k} x-\lambda_{k}(x)\right)$ may not exist.

If there is no gap between the blocks $I_{k}$, i.e. $\cup_{k=1}^{\infty} I_{k}=\mathbb{N}$ and $\left|I_{k}\right| \uparrow \infty$, then Abel rearrangement shows that $\sum_{k=1}^{N} \lambda_{k}(x)=O(1)$, and thus in (7.2) the centering factor $\lambda_{k}(x)$ can be omitted. For a motivation of (7.2) and the centering factors $\lambda_{k}(x)$, let us note that for any fixed $x \in \mathbb{R}$ the law of the iterated logarithm for independent bounded r.v.'s implies

$$
\limsup _{N \rightarrow \infty} \frac{\sum_{k=1}^{N}\left(\sin n_{k} x-\lambda_{k}(x)\right)}{\left(2 \gamma_{N}^{* 2}(x) \log \log \gamma_{N}^{* 2}(x)\right)^{1 / 2}}=1 \quad \mathbb{P}-\text { a.s. }
$$

where

$$
\gamma_{N}^{* 2}(x)=\sum_{k=1}^{N} \mathbb{E}\left(\sin n_{k} x-\lambda_{k}(x)\right)^{2} \sim \gamma_{N}^{2}(x)
$$


and the last relation follows from the strong law of large numbers. Clearly, (7.6) and (7.7) yield for any fixed $x \in \mathbb{R}$

$$
\limsup _{N \rightarrow \infty} \frac{\sum_{k=1}^{N}\left(\sin n_{k} x-\lambda_{k}(x)\right)}{\left(2 \gamma_{N}^{2}(x) \log \log \gamma_{N}^{2}(x)\right)^{1 / 2}}=g(x) \quad \mathbb{P}-\text { a.s. }
$$

By Fubini's theorem, with $\mathbb{P}$-probability 1, (7.8) holds for almost all $x \in(0,2 \pi)$ providing the LIL corresponding to (7.2). Of course, Fubini's theorem cannot be applied for distributional limit theorems like the CLT (and in case of the CLT, the factor $g(x)$ in the denominator cannot be brought to the right hand side) and the proof of (7.5) requires an elaborate argument.

We pass now to the study of non-centered partial sums $S_{N}=\sum_{k=1}^{N} \sin n_{k} x$. In contrast to $S_{N}-\mathbb{E} S_{N}$, the behavior of $S_{N}$ depends on the size of the gaps $\Delta_{k}$ : for $\Delta_{k}=0$ the limit distribution of $S_{N} / \sqrt{N}$ is mixed Gaussian with $\mathbb{P}$-probability 1 by a result of Bobkov and Götze [21] and for exponentially growing $\Delta_{k}$ the SalemZygmund CLT implies that $S_{N} / \sqrt{N} \stackrel{d}{\longrightarrow} N(0,1 / 2)$. Our next result shows that in regular cases $\left(S_{N}-\mathbb{E} S_{N}\right) / \sqrt{N}$ and $\mathbb{E} S_{N} / \sqrt{N}$ are asymptotically independent, reducing the behavior of $S_{N} / \sqrt{N}$ to that of the nonrandom trigonometric sum $\mathbb{E} S_{N} / \sqrt{N}$.

Theorem 7.2 Let $I_{1}, I_{2}, \ldots$ be disjoint intervals of positive integers such that the sets $\left\{k \in \mathbb{N}:\left|I_{k}\right|=d\right\}, d=1,2, \ldots$ have asymptotic densities $\mu_{d}$. Let $n_{1}, n_{2}, \ldots$ be independent random variables defined on a probability space $(\Omega, \mathcal{A}, \mathbb{P})$ such that $n_{k}$ is uniformly distributed on $I_{k}$. Let $\lambda_{k}(x)$ and $g(x)$ be defined by (7.1) and (7.4). Then $\mathbb{P}$-almost surely

$$
\frac{1}{\sqrt{N g(x)}} \sum_{k=1}^{N}\left(\sin n_{k} x-\lambda_{k}(x)\right) \stackrel{d}{\longrightarrow} N(0,1 / 2)
$$

and

$$
\frac{1}{\sqrt{N}} \sum_{k=1}^{N}\left(\sin n_{k} x-\lambda_{k}(x)\right) \stackrel{d}{\longrightarrow} F
$$

with respect to the probability space $((0,2 \pi), \mathcal{B}, \lambda)$, where $F$ is the mixed Gaussian distribution with characteristic function

$$
\phi(\lambda)=\int_{0}^{2 \pi} \exp \left(-\frac{\lambda^{2}}{2} g(x)\right) d x .
$$

If in addition we have

$$
\frac{1}{\sqrt{N}} \sum_{k=1}^{N} \lambda_{k}(x) \stackrel{d}{\longrightarrow} G
$$

with respect to to any interval $E \subset(0,2 \pi)$ with positive measure, then $\mathbb{P}$-almost surely

$$
\left(\frac{1}{\sqrt{N}} \sum_{k=1}^{N}\left(\sin n_{k} x-\lambda_{k}(x)\right), \frac{1}{\sqrt{N}} \sum_{k=1}^{N} \lambda_{k}(x)\right) \stackrel{d}{\longrightarrow}(F, G)
$$

where the components of the limit vector are independent. 
Theorem 7.2 shows that $S_{N}^{(1)}=S_{N}-\mathbb{E} S_{N}$ and $S_{N}^{(2)}=\mathbb{E} S_{N}$ are asymptotically independent and thus we can study their contributions separately. Note that $S_{N}^{(2)}$, the averaged version of $S_{N}$, is a nonrandom trigonometric sum. In the case when $\left|I_{k}\right|=d$ for all $k$, we have

$$
\lambda_{k}(x)=\mathbb{E}\left(\sin n_{k} x\right)=d^{-1} \sum_{j \in I_{k}} \sin j x=\frac{\sin (d x / 2)}{d \sin (x / 2)} \sin \left(A_{k}+d / 2+1 / 2\right) x
$$

where $A_{k}$ is the smallest integer of $I_{k}$ and thus

$$
S_{N}^{(2)}=\frac{\sin (d x / 2)}{d \sin (x / 2)} \sum_{k=1}^{N} \sin \left(A_{k}+d / 2+1 / 2\right) x .
$$

As one can show, if the gaps $\Delta_{k}=A_{k+1}-A_{k}-d$ between the intervals remain constant or if the $A_{k}$ are integers and $\Delta_{k} \uparrow \infty, \Delta_{k}=O\left(k^{\gamma}\right)$ with $\gamma<1 / 4$ (small gaps), then (7.2) holds with $\lambda_{k}=0$, i.e. without a centering factor. At the other end of the spectrum, i.e. for rapidly increasing $A_{k}$, the centering factors themselves contribute to the limit distribution, i.e.

$$
\frac{1}{\sqrt{N}} \sum_{k=1}^{N} \lambda_{k}(x)
$$

has a nondegenerate limit distribution. More precisely, if $A_{k}$ satisfies the Erdős gap condition

$$
A_{k+1} / A_{k} \geq 1+c_{k} / \sqrt{k}, \quad c_{k} \rightarrow \infty
$$

then (7.15) has the limit distribution with characteristic function

$$
\phi(\lambda)=\frac{1}{2 \pi} \int_{0}^{2 \pi} \exp \left(-\frac{\lambda^{2}}{4} \cdot \frac{\sin ^{2}(d x / 2)}{d^{2} \sin ^{2}(x / 2)}\right) d x
$$

and thus by the asymptotic independence of the components of (7.13) it follows that

$$
N^{-1 / 2} \sum_{k=1}^{N} \sin n_{k} x
$$

has a pure Gaussian limit distribution $N(0,1 / 2)$. Since in this case $\left(n_{k}\right)$ also satisfies the analogue of (7.16), the asymptotic normality of (7.18) follows from Erdős' central limit theorem [26] even for nonrandom $\left(n_{k}\right)$, i.e. in this case Theorem 7.2 reduces to a result in classical lacunarity theory. It is interesting to note that in this case the pure Gaussian limit distribution of $S_{N} / \sqrt{N}$ is obtained as the convolution of two mixed Gaussian distributions. In the intermediate case between slowly and rapidly increasing $\left(n_{k}\right)$, the centering factors $\lambda_{k}(x)$ in (7.1) may or may not contribute to the limit distribution $F$ and $F$ may be nongaussian. In view of (7.14), from the results of Berkes [12] it follows that there exist sequences $\left(A_{k}\right)$ satisfying (7.16) with $c_{k} \rightarrow \infty$ replaced by $c_{k}=c>0$ such that (7.15) has a nongaussian limit distribution and for any positive sequence $c_{k} \rightarrow 0$ there exist sequences $\left(A_{k}\right)$ satisfying (7.16) such 
that (7.15) tends to 0 in probability. This shows that nongaussian limits of (7.15) can occur arbitrary close to the gap condition (7.16), i.e. (7.16) is critical in the theory. Theorem 7.2 also shows that the limit distribution of (7.18), if exists, is the convolution of a mixed normal distribution and the limit distribution of a normed trigonometric sum with nonrandom frequencies $A_{k}+(d+1) / 2$. The asymptotic behavior of such nonrandom sums is an arithmetic rather than a probabilistic problem and we do not discuss it here.

For the proof of the results in this chapter we refer to Berkes and Raseta [14].

\section{References}

[1] C. Aistleitner and I. Berkes. On the central limit theorem for $f\left(n_{k} x\right)$. Prob. Theory Rel. Fields 146 (2010), 267-289.

[2] C. Aistleitner, I. Berkes and R. Tichy. On permutations of HardyLittlewood-Pólya sequences. Trans Amer. Math. Soc. 363 (2011) 6219-6244.

[3] C. Aistleitner, I. Berkes and R. Tichy. On permutations of lacunary series. RIMS Kokyuroku Bessatsu B34 (2012), 1-25.

[4] C. Aistleitner, I. Berkes and R. Tichy. On the system $f(n x)$ and probabilistic number theory. Anal. Probab. Methods Number Theory, E. Manstavicius et al., Vilnius 2012, 1-18.

[5] C. Aistleitner, I. Berkes and R. Tichy. On the law of the iterated logarithm for permuted lacunary sequences. Proceedings of the Steklov Institute of Mathematics 276 (2012), 3-20.

[6] C. Aistleitner, I. Berkes and R. Tichy. On the asymptotic behavior of weakly lacunary sequences. Proc. Amer. Math. Soc. 139 (2011) 2505-2517.

[7] D. J. Aldous. Limit theorems for subsequences of arbitrarily-dependent sequences of random variables, $Z$. Wahrscheinlichkeitstheorie verw. Gebiete $\mathbf{4 0}$ (1977), 59-82.

[8] D. J. Aldous. Subspaces of $L_{1}$ via random measures. Trans. Amer. Math. Soc. 267 (1981), 445-463.

[9] F. Amoroso and E. Viada, Small points on subvarieties of a torus. Duke Math. J. 150(3), 407-442 (2009).

[10] I. Berkes. A central limit theorem for trigonometric series with small gaps, Zeitschrift für Wahrscheinlichkeitstheorie verw. Gebiete 47 (1979), 157-161.

[11] I. Berkes. On almost symmetric sequences in $L_{p}$. Acta Math. Hung. 54 (1989) 269-278.

[12] I. Berkes. Nongaussian limit distributions of lacunary trigonometric series. Canad. J. Math. 43 (1991) 948-959.

[13] I. BERKES and E. PÉTER. Exchangeable random variables and the subsequence principle, Prob. Theory Rel. Fields 73 (1986), 395-413. 
[14] I. Berkes and M. Raseta. On trigonometric sums with random frequencies. Preprint.

[15] I. Berkes and H. P. Rosenthal. Almost exchangeable sequences of random variables, Z. Wahrscheinlichkeitstheorie verw. Gebiete 70 (1985), 473-507.

[16] I. Berkes and R. Tichy. On permutation-invariance of limit theorems. J. Complexity 31 (2015) 372-379.

[17] I. Berkes and R. Tichy. Lacunary series and stable distributions. In: Mathematical statistics and limit theorems. Festschrift for P. Deheuvels. M. Hallin, D.M. Mason, D. Pfeifer, J. Steinebach (eds.) Springer, 2015, pp. 7-19.

[18] I. Berkes and R. Tichy. The Kadec-Pełczynski theorem in $L^{p}, 1 \leq p<2$. Proc. Amer. Math. Soc. 144 (2016), 2053-2066.

[19] I. Berkes and R. Tichy. Resonace theorems and the central limit theorem for lacunary series. Preprint.

[20] I. Berkes and R. Tichy. A uniform version of the subsequence principle. Preprint.

[21] S. Bobkov and F. Götze, Concentration inequalities and limit theorems for randomized sums. Probab. Theory Related Fields 137 (2007), 49-81.

[22] S. D. Chatterji, A general strong law. Invent. Math. 9 (1970), 235-245.

[23] S. D. ChatterJi. A principle of subsequences in probability theory: The central limit theorem. Adv. Math. 13 (1974), 31-54.

[24] S. D. ChatterJi. A subsequence principle in probability theory II. The law of the iterated logarithm. Invent. Math. 25 (1974), 241-251.

[25] S. D. Chatteru. Un principe de sous-suites dans la théorie des probabilités. Séminaire des probabilités VI, Strasbourg, Lecture Notes in Mathematics 258, pp. 72-89. Springer, 1972.

[26] P. ERDős. On trigonometric sums with gaps. Magyar Tud. Akad. Mat. Kut. Int. Közl. 7 (1962), 37-42.

[27] P. ERdős and I.S. GÁL. On the law of the iterated logarithm. Proc. Nederl. Akad. Wetensch. Ser A 58, 65-84, 1955.

[28] J.-H. Evertse, R. H.-P. Schlickewei and W. M. Schmidt. Linear equations in variables which lie in multiplicative group, Ann. of Math. 155 (2002), no. $3,807-836$.

[29] K. Fukuyama. The law of the iterated logarithm for the discrepancies of a permutation of $\left\{n_{k} x\right\}$. Acta Math. Acad. Sci. Hung. 123 (2009), 121-125.

[30] K. Fukuyama and B. Petit. Le théorème limite central our les suites de R. C. Baker. Ergodic Theory Dynamic Systems 21, 479-492 (2001)

[31] J. Galambos. The asymptotic theory of extreme order statistics. Robert E. Krieger Publishing Co., Melbourne, FL, second edition, 1987.

[32] V. F. Gaposhkin. Lacunary series and independent functions. Russian Math. Surveys 21 (1966), 1-82. 
[33] V. F. Gaposhkin, The central limit theorem for some weakly dependent sequences. Theory Probab. Appl. 15 (1970), 649-666.

[34] V. F. Gaposhkin. On some systems of almost independent functions, Siberian Math. J. 9 (1968), 198-210.

[35] A. Garsia. Existence of almost everywhere convergent rearrangements for Fourier series of $L_{2}$ functions. Ann. Math. 79 (1964), 623-629.

[36] S. Guerre. Types and suites symétriques dans $L^{p}, 1 \leq p<+\infty$, Israel J. Math.

[37] M.I. Kadec and W. Peeczyński. Bases, lacunary sequences and complemented subspaces in the spaces $L_{p}$. Studia Math. 21 1961/1962, 161-176.

[38] M. KAC, On the distribution of values of sums of the type $\sum f\left(2^{k} t\right)$. Ann. of Math. 47 (1946), 33-49.

[39] M. KaC. Probability methods in some problems of analysis and number theory, Bull. Amer. Math. Soc. 55 (1949), 641-665.

[40] J. Komlós. A generalization of a problem of Steinhaus. Acta Math. Acad. Sci. Hungar. 18, 217-229, (1967)

[41] J. KomLós. Every sequence converging to 0 weakly in $L_{2}$ contains an unconditional convergence sequence, Ark. Math. 12 (1974), 41-49.

[42] D.E. Menshov. Sur la convergence et la sommation des séries de fonctions orthogonales, Bull. Soc. Math. France 64 (1936), 147-170.

[43] W. Morgenthaler. A central limit theorem for uniformly bounded orthonormal systems. Trans. Amer. Math. Soc. 79, (1955), 281-311.

[44] R. NAIR. On strong uniform distribution. Acta Arith. 56 (1990), 183-193.

[45] E. M. Nikishin. Resonance theorems and superlinear operators. Russian Math. Surveys 25/6 (1970), 125-187.

[46] R. RAnga RaO. Relations between weak and uniform convergence of measures with applications, Ann. Math. Statist, 33 (1962), 659-680.

[47] R. Salem and A. Zygmund. On lacunary trigonometric series, Proc. Nat. Acad. Sci. USA 33 (1947), 333-338.

[48] S. Takahasi. On lacunary trigonometric series, Proc, Japan Acad. 41 (1965), $503-506$.

[49] S. TAKAhASHI. On the law of the iterated logarithm for lacunary trigonometric series. Tohoku Math. J. 24 (1972), 319-329.

[50] R. Tijdemann. On integers with many small prime factors, Compositio Math. 26 (1973), 319-330.

[51] P. Uluanov. Solved and unsolved problems in the theory of trigonometric and orthogonal series. (Russian). Uspehi Mat. Nauk 19/1 (1964), 1-69.

[52] M. Weiss. On the law of the iterated logarithm for uniformly bounded orthonormal systems. Trans. Amer. Math. Soc. 92 (1959) 531-553.

[53] A. Zygmund, Trigonometric series. Vol. I, II. Third edition. Cambridge University Press. 2002. 